University of Texas at El Paso

ScholarWorks@UTEP

4-2017

\title{
It Is Possible to Determine Exact Fuzzy Values Based on an Ordering of Interval-Valued or Set-Valued Fuzzy Degrees
}

\author{
Gerardo Muela \\ The University of Texas at El Paso, gdmuela@miners.utep.edu \\ Olga Kosheleva \\ The University of Texas at El Paso, olgak@utep.edu \\ Vladik Kreinovich \\ The University of Texas atEl Paso, vladik@utep.edu
}

Follow this and additional works at: https://scholarworks.utep.edu/cs_techrep

Part of the Computer Sciences Commons

Comments:

Technical Report: UTEP-CS-17-11a

\section{Recommended Citation}

Muela, Gerardo; Kosheleva, Olga; and Kreinovich, Vladik, "It Is Possible to Determine Exact Fuzzy Values Based on an Ordering of Interval-Valued or Set-Valued Fuzzy Degrees" (2017). Departmental Technical Reports (CS). 1107.

https://scholarworks.utep.edu/cs_techrep/1107

This Article is brought to you for free and open access by the Computer Science at ScholarWorks@UTEP. It has been accepted for inclusion in Departmental Technical Reports (CS) by an authorized administrator of ScholarWorks@UTEP.For more information, please contact Iweber@utep.edu. 


\section{It Is Possible to Determine Exact Fuzzy Values Based on an Ordering of Interval-Valued or Set-Valued Fuzzy Degrees}

\author{
Gerardo Muela, Olga Kosheleva, and Vladik Kreinovich \\ University of Texas at El Paso \\ $500 \mathrm{~W}$. University \\ El Paso, TX 79968, USA \\ gdmuela@miners.utep.edu, olgak@utep.edu \\ vladik@utep.edu
}

\author{
Christian Servin \\ Computer Science and \\ Information Technology Systems Department \\ El Paso Community College \\ 919 Hunter, El Paso, TX 79915, USA \\ cservin@gmail.com
}

\begin{abstract}
In the usual [0,1]-based fuzzy logic, the actual numerical value of a fuzzy degree can be different depending on a scale, what is important - and scale-independent - is the order between different values. To make a description of fuzziness more adequate, it is reasonable to consider interval-valued degrees instead of numerical ones. Here also, what is most important is the order between the degrees. If we have only order between the intervals, can we, based on this order, reconstruct the original numerical values - i.e., the degenerate intervals? In this paper, we show that such a reconstruction is indeed possible, moreover, that it is possible under three different definitions of order between numerical values.
\end{abstract}

\section{Formulation of the Problem}

Need for probabilities and need for fuzzy degrees. To describe how frequently different events occur, a natural idea is to use probabilities - i.e., in effect, frequencies with which this event has occurred. For example, if under certain weather conditions, in the past, rain happens in $30 \%$ of the cases, we say that under these conditions, the probability of rain is $30 \%$.

In general, if out of $n$ cases, the event of interest happened in $m$ of them, we say that the probability of the event is equal to $m / n$. Strictly speaking, this frequency is only an approximation to the actual probability; the larger the sample size $n$, the more accurate this approximation. So, if we want a more accurate estimate for the probability, we need to increase $n$, i.e., to consider a larger sample.

In addition to this objective probability, there is also subjective uncertainty: experts are not $100 \%$ sure about their statements. For example, an expert may say that the probability of rain under certain conditions is small, without providing a precise numerical description of what this word means.

To process this subjective uncertainty, we need to describe in computer-understandable terns, i.e., in terms of numbers. This description is one of the main objectives of fuzzy logic; see, e.g., [2], [5], [10]. To describe an imprecise ("fuzzy") word like "small" in precise terms, we can, e.g., for each possible value $x$ of the corresponding quantity (in the above example, for each possible probability value) to estimate, on a given scale, how certain this expert is that $x$ is small. If the expert marked his or her degree of certainty as 7 on a scale from 0 to 10 , we can then say that the expert's degree of certainty is $7 / 10$. In general, if an expert marked $m$ on a scale from 0 to $n$, we can use the value $m / n$.

An important difference between probabilities and fuzzy degrees. In both cases of probabilistic and fuzzy uncertainty, we have the same formula $m / n$ for estimating the corresponding degree. However, there is a big difference between these values.

Probabilities are objective. If two people use the same data, they will get the same probability value.

In contrast, expert opinions are subjective. Based on the same evidence, and based on the same understanding of what is more probable and what is less probable, some experts will be more "optimistic" and mostly use values close to 10 on a scale from 0 to 10 , while other experts may be more "pessimistic" and mostly use values close to 0 on the same scale. In this case, the values $m / n$ corresponding to one expert can be transformed into values $m^{\prime} / n^{\prime}$ corresponding to another expert by an appropriate non-linear re-scaling.

As a result, in fuzzy logic, the actual numerical value of a fuzzy degree can be different depending on a scale. What is important - and scale-independent - is the order between different values.

Need for interval-valued fuzzy logic. Fuzzy logic deals with situations in which an expert uses imprecise words like "small" to describe his or her opinion. The expert uses imprecise words because he or she is unable to come up with an exact estimate.

On the other hand, the traditional [0,1]-based fuzzy logic requires the same expert to come up with an exact value of a scale from 0 to 10 that describes this expert's degree of certainty. Of course, in practice, the expert is often unable to do it. To be more precise, the expect may be able to confidently say that his.her degree of certainty is 7 and not 6 and not 8, but if we try to get a more accurate description by taking a scale form, say, 1 to 100 , it is doubtful that the expert will be able to mark his/her degree of confidence as 71 and not 
70 or 72 . At best, the expert would be able to mark a whole interval of possible values - e.g., from 65 to 75 - as describing his/her degree of certainty. This corresponds to the interval $[0.65,0.75]$ of possible degree.

Such interval-valued fuzzy techniques have indeed been proposed. They are indeed more adequate in describing expert's uncertainty, and they have led to many practical applications; see, e.g., [3], [4], [8].

Interval values generalize the usual fuzzy logic: each degree $a \in[0,1]$ from the original fuzzy logic can also be viewed as a "degenerate" interval $[a, a]$ in the interval-valued fuzzy scheme - but, of course, in interval-valued approach, we have additional degrees $[a, b]$ with $a<b$.

Formulation of the problem. In the interval-valued case, also re-scalings are possible. As a result, what is most important is the numerical values, but rather the order between the degrees.

If we have only order between the intervals, can we, based on this order, reconstruct the original numerical values - i.e., the degenerate intervals?

What we do in this paper. In this paper, we show that such a reconstruction is indeed possible, moreover, that it is possible under three different definitions of order between numerical values.

\section{First ORdering: LATTICE (COMPONENT-Wise) ORDER}

Component-wise order between intervals: a brief reminder. If for some statement, the expert's degree of confidence is represented by an interval $[a, b]$, and then we increase the lower bound, to make the interval $\left[a^{\prime}, b\right]$ with $a^{\prime}>a$, we thus increase our degree of confidence in this statement. Similarly, if we increase $b$ to $b^{\prime}>b$, we thus increase our degree of confidence. From this viewpoint, it makes sense to say that the interval $\left[a^{\prime}, b^{\prime}\right]$ represents a larger (or same) degree of confidence than the interval $[a, b]$ if $a^{\prime} \geq a$ and $b^{\prime}>b$ :

$$
[a, b] \leq\left[a^{\prime}, b^{\prime}\right] \Leftrightarrow\left(a \leq a^{\prime} \& b \leq b^{\prime}\right)
$$

Another way to describe the component-wise order. Let us show that the above order between intervals $I=[a, b]$ and $I^{\prime}=\left[a^{\prime}, b^{\prime}\right]$ can be described in the following equivalent way:

$$
\forall x \in I \exists x^{\prime} \in I^{\prime}\left(x \leq x^{\prime}\right) \& \forall x^{\prime} \in I^{\prime} \exists x \in I\left(x \leq x^{\prime}\right) .
$$

Indeed, if this property is true, then, since the first part of it is true for all $x \in I=[a, b]$, in particular, it is true for $x=b$. Thus, for $x=b$, there exists a value $x^{\prime} \in I^{\prime}=\left[a^{\prime}, b^{\prime}\right]$ for which $b=x \leq x^{\prime}$. From $b \leq x^{\prime}$ and $x^{\prime} \leq b^{\prime}$, we conclude that $b \leq b^{\prime}$.

Similarly, from the second part of the above property, for $x^{\prime}=a^{\prime}$, we conclude that $x \leq x^{\prime}=a^{\prime}$ for some $x \in[a, b]$. From $a \leq x$ and $x \leq a^{\prime}$, we conclude that $a \leq a^{\prime}$. Thus, the above definition implies component-wise order between intervals.

Vice versa, if $I \leq I^{\prime}$ in the sense of the component-wise order, then, as is easy to show, we can always take $x^{\prime}=b^{\prime}$ in the first part of the property and $b=x$ for the second part. The equivalence is proven.

Formulation of the problem in precise terms. Suppose that on the set of all subintervals $[a, b]$ of the interval $[0,1]$, we have the above ordering.

Based on this ordering, can we uniquely determine degenerate intervals, i.e., intervals of the type $[a, a]$ ? In this section, we will answer that this is indeed possible. This determination will be done step by step.

First step: it is possible to define the interval $[0,0]$ based only on the order. Indeed, $[0,0]$ is the only interval which is smaller (in the sense of the above relation $\leq$ ) than any other interval.

In precise terms, the interval $[0,0]$ is the only interval $I$ that satisfies the property

$$
\forall J(I \leq J),
$$

where variables $I$ and $J$ go over intervals.

Second step: it is possible to defined intervals of the type $[0, a]$ based only on the order. Our claim is that an interval $I$ is of type $[0, a]$ if and only if the set of all intervals between $[0,0]$ and $I$ is linearly ordered, i.e., if and only if

$$
\begin{gathered}
\forall J \forall J^{\prime}\left(\left([0,0] \leq J \leq I \&[0,0] \leq J^{\prime} \leq I\right) \Rightarrow\right. \\
\left.\left(J \leq J^{\prime} \vee J^{\prime} \leq J\right)\right) .
\end{gathered}
$$

Indeed, if $I=[0, a]$, then for each interval $J=[b, c]$, the condition

$$
[0,0] \leq J=[b, c] \leq[0, a]
$$

implies that $0 \leq b \leq 0$ and thus, that $b=0$. So, all such intermediate intervals $J$ and $J^{\prime}$ have the form $[0, c]$ for some real value $c$.

Of course, all such intervals are linearly ordered. Indeed, for $J=[0, c]$ and $J^{\prime}=\left[0, c^{\prime}\right]$, either $c \leq c^{\prime}$ or $c^{\prime} \leq c$.

- In the first case, we have $J \leq J^{\prime}$.

- In the second case, we have $J^{\prime} \leq J$.

Let us show that, vice versa, if the interval $I$ has the form $[a, b]$ with $a \neq 0$ (i.e., $a>0$ ), then there exist $J$ and $J^{\prime}$ between $[0,0]$ and $I$ for which $J \not \leq J^{\prime}$ and $J^{\prime} \not \leq J$. Indeed, it is sufficient to take $J=[a, 0]$ and $J^{\prime}=[0, b]$.

Final step: it is possible to define degenerate intervals based only on the order. We already know how to define a degenerate interval $[0,0]$.

Our claim is that $I$ is a degenerate interval $[a, a]$, with $a>0$ if and only if $I$ is not of the type $[0, a]$ and there exists an interval $I^{\prime}$ of the type $[0, a]$ for which

- $I^{\prime} \leq I$,

- the set of all intervals $J$ between $I^{\prime}$ and $I$ is linearly ordered, and

- for no larger interval $I^{\prime \prime} \geq I, I^{\prime \prime} \neq I$, the set of all intervals $J$ between $I^{\prime}$ and $I^{\prime \prime}$ is linearly ordered.

Indeed, if $I=[a, a]$ for some $a>0$, then we can take $I^{\prime}=[0, a]$ for this same $a$. Then all intervals $J$ and $J^{\prime}$ between 
$I^{\prime}$ and $I$ have the form $[b, a]$ for some $b$ and the same $a$ and are, thus, linearly ordered.

On the other hand, if $I^{\prime \prime}=\left[a^{\prime \prime}, b^{\prime \prime}\right]$ is larger than $I=[a, a]$, this means that either $a^{\prime \prime}>a-$ in which case $b^{\prime \prime} \geq a^{\prime \prime}>a$ and thus $b^{\prime \prime}>a-$ or $b^{\prime \prime}>a$. Then, both $J=I$ and $J^{\prime}=\left[0, b^{\prime \prime}\right]$ are between $I^{\prime}$ and $I^{\prime \prime}$, but $J \not \leq J^{\prime}$ and $J^{\prime} \not \leq J$.

Vice versa, let us assume that $I$ is a non-degenerate interval $\left[a_{0}, b_{0}\right]$ for some $a_{0}<b_{0}$. the fact that this is not an interval of type $[0, a]$ means that $a_{0}>0$. In this case, linear ordering for all intervals $J$ and $J^{\prime}$ between $I^{\prime}=[0, a]$ and $I=\left[a_{0}, b_{0}\right]$ is only possible if $b_{0}=a$. Indeed, if $b_{0}=a$, then we do get the linear ordering, but if $b_{0}>a$, then the intervals $J=\left[0, b_{0}\right]$ and $J^{\prime}=\left[a_{0}, a\right]$ are between $I^{\prime}$ and $I$, but $J \not \leq J^{\prime}$ and $J^{\prime} \leq J$.

So, if there is a linear ordering of all intervals between $I^{\prime}$ and $I$, then $b_{0}=a$, and the interval $I$ has the form $\left[a_{0}, a\right]$, with $a_{0}<a$. However, now we can take a larger interval $I^{\prime \prime}=[a, a] \geq I$, and still be able to conclude that all intervals between $I^{\prime}$ and $I^{\prime \prime}$ are linearly ordered - which contradicts to our requirement that no such larger interval is possible.

Thus, the above condition indeed uniquely determines degenerate intervals.

\section{SECOND ORDERING: NECESSARILY LARGER}

Description of the "necessarily larger" ordering. The fact that for a statement $S$, instead of a single fuzzy value we have an interval $[a, b]$ of possible fuzzy values can be interpreted as saying that the actual (unknown) expert's degree of confidence in this statement can be any value between $a$ and $b$.

Similarly, for another statement $S^{\prime}$, the corresponding interval $\left[a^{\prime}, b^{\prime}\right]$ means that the actual (unknown) expert's degree of confidence in the statement $S^{\prime}$ can be any value between $a^{\prime}$ and $b^{\prime}$.

A reasonable idea is to ask when we can be absolutely certain that our degree of belief in $S$ is smaller than or equal to the degree of belief in $S^{\prime}$. Since we only know that the intervals that contain the actual values, the only way to be absolutely certain is to make sure that each value from the interval $[a, b]$ is smaller than or equal to any value from the interval $\left[a^{\prime}, b^{\prime}\right]$.

This means, in particular, that $b \leq a^{\prime}$, Vice versa, if $b \leq a^{\prime}$, then any value from the interval $[a, b]$ is smaller than or equal to $b$ and is, thus, smaller than or equal to $a^{\prime}$. In its turn, $a^{\prime}$ is smaller than or equal to any value from the interval $\left[a^{\prime}, b^{\prime}\right]$. Thus, indeed, if $b \leq a^{\prime}$, then any value from the interval $[a, b]$ is smaller than or equal to any value from the interval $\left[a^{\prime}, b^{\prime}\right]$, So, the "necessarily larger" relation takes the following form:

$$
[a, b] \leq\left[a^{\prime}, b^{\prime}\right] \Leftrightarrow b \leq a^{\prime} .
$$

Formulation of the problem in precise terms. Suppose that on the set of all subintervals $[a, b]$ of the interval $[0,1]$, we have the above ordering.

Based on this ordering, can we uniquely determine degenerate intervals, i.e., intervals of the type $[a, a]$ ? In this section, we will answer that this is indeed possible. This determination will be also done step by step.
First step: it is possible to describe interval inclusion based only on the order. Let us first show that the notion of interval inclusion $[a, b] \subseteq\left[a^{\prime}, b^{\prime}\right]$ can be described based only on the above-defined order. Namely, we will show that

$$
\begin{gathered}
I \subseteq I^{\prime} \Leftrightarrow \\
\forall I^{\prime \prime}\left(\left(I^{\prime} \leq I^{\prime \prime} \Rightarrow I \leq I^{\prime \prime}\right) \&\left(I^{\prime \prime} \leq I^{\prime} \Rightarrow I^{\prime \prime} \leq I\right)\right) .
\end{gathered}
$$

Indeed, let us assume that $I \subseteq I^{\prime}$. For every interval $I^{\prime \prime}$, the relation $I^{\prime} \leq I^{\prime \prime}$ means that every element from the interval $I^{\prime}$ is smaller than or equal to every element from the interval $I^{\prime \prime}$. Since $I \subseteq I^{\prime}$, every element of $I$ is also an element of $I^{\prime}$ and is, thus, smaller than or equal to every element of $I^{\prime \prime}$. This means that $I \leq I^{\prime \prime}$.

Similarly, for every interval $I^{\prime \prime}$, the relation $I^{\prime \prime} \leq I^{\prime}$ means that every element from the interval $I^{\prime \prime}$ is smaller than or equal to every element from the interval $I^{\prime}$. Since $I \subseteq I^{\prime}$, every element of $I$ is also an element of $I^{\prime}$ and is, thus, larger than or equal to every element of $I^{\prime \prime}$. This means that $I^{\prime \prime} \leq I$.

Vice versa, let us assume that for $I=[a, b]$ and $I^{\prime}=\left[a^{\prime}, b^{\prime}\right]$, we have

$$
\forall I^{\prime \prime}\left(\left(I^{\prime} \leq I^{\prime \prime} \Rightarrow I \leq I^{\prime \prime}\right) \&\left(I^{\prime \prime} \leq I^{\prime} \Rightarrow I^{\prime \prime} \leq I\right)\right)
$$

In particular, for $I^{\prime \prime}=\left[b^{\prime}, b^{\prime}\right]$, we have $I^{\prime} \leq I^{\prime \prime}$ and thus, we have $I \leq I^{\prime \prime}$, i.e., $[a, b] \leq\left[b^{\prime}, b^{\prime}\right]$. According to our description of the "necessarily larger" relation, this means that $b \leq b^{\prime}$.

Similarly, for $I^{\prime \prime}=\left[a^{\prime}, a^{\prime}\right]$, we have $I^{\prime \prime} \leq I^{\prime}$ and thus, we have $I^{\prime \prime} \leq I$, i.e., $\left[a^{\prime}, a^{\prime}\right] \leq[a, b]$. According to our description of the "necessarily larger" relation, this means that $a^{\prime} \leq a$.

So, $a^{\prime} \leq a \leq b \leq b^{\prime}$, which means exactly that $[a, b] \subseteq$ $\left[a^{\prime}, b^{\prime}\right]$

Final step: it is possible to define degenerate intervals based only on the order. We already know how to define inclusion in terms of the order.

A degenerate interval $I$ can then be defined as the one that does not have any subinterval different from itself:

$$
\forall J(J \subseteq I \Rightarrow J=I) .
$$

\section{Third ORDERING: POSSIBLy LARGER}

Description of the "possibly larger" ordering. Another reasonable idea is to ask when it is possible that our degree of belief in $S$ is smaller than or equal to the degree of belief in $S^{\prime}$. Since we only know that the intervals that contain the actual values, this means that there exists a value from the interval $[a, b]$ which is smaller than or equal to some value from the interval $\left[a^{\prime}, b^{\prime}\right]$.

If $v \leq v^{\prime}$ for some $v$ and $v^{\prime}$ for which $a \leq v \leq b$ and $a^{\prime} \leq v^{\prime} \leq b^{\prime}$, then from $a \leq v \leq v^{\prime} \leq b^{\prime}$, we conclude that $a \leq b^{\prime}$.

Vice versa, if $a \leq b^{\prime}$, then we have values $a \in[a, b]$ and $b^{\prime} \in$ $\left[a^{\prime}, b^{\prime}\right]$ for which $a \leq b^{\prime}$ and thus, $[a, b]$ is possibly smaller than $\left[a^{\prime}, b^{\prime}\right]$. So, the "possibly larger" relation takes the following form:

$$
[a, b] \leq\left[a^{\prime}, b^{\prime}\right] \Leftrightarrow a \leq b^{\prime}
$$


Formulation of the problem in precise terms. Suppose that on the set of all subintervals $[a, b]$ of the interval $[0,1]$, we have the above ordering.

Based on this ordering, can we uniquely determine degenerate intervals, i.e., intervals of the type $[a, a]$ ? In this section, we will answer that this is indeed possible. This determination - similarly to the two previous cases - will be done step by step.

First step: it is possible to describe interval inclusion based only on the order. Let us first show that the notion of interval inclusion $[a, b] \subseteq\left[a^{\prime}, b^{\prime}\right]$ can be described based only on the above-defined order. Namely, we will show that

$$
\begin{gathered}
I \subseteq I^{\prime} \Leftrightarrow \\
\forall I^{\prime \prime}\left(\left(I \leq I^{\prime \prime} \Rightarrow I^{\prime} \leq I^{\prime \prime}\right) \&\left(I^{\prime \prime} \leq I \Rightarrow I^{\prime \prime} \leq I^{\prime}\right)\right) .
\end{gathered}
$$

Indeed, let us assume that $I \subseteq I^{\prime}$. For every interval $I^{\prime \prime}$, the relation $I \leq I^{\prime \prime}$ means that some element from the interval $I$ is smaller than or equal to some element from the interval $I^{\prime \prime}$. Since $I \subseteq I^{\prime}$, every element of $I$ is also an element of $I^{\prime}$. Thus, some element of $I^{\prime}$ is smaller than or equal than some element of $I^{\prime \prime}$. This means that $I^{\prime} \leq I^{\prime \prime}$.

Similarly, for every interval $I^{\prime \prime}$, the relation $I^{\prime \prime} \leq I$ means that some element from the interval $I^{\prime \prime}$ is smaller than or equal to some element from the interval $I$. Since $I \subseteq I^{\prime}$, every element of $I$ is also an element of $I^{\prime}$. Thus, some element of $I^{\prime \prime}$ is smaller than or equal than some element of $I^{\prime}$. This means that $I^{\prime \prime} \leq I^{\prime}$.

Vice versa, let us assume that for $I=[a, b]$ and $I^{\prime}=\left[a^{\prime}, b^{\prime}\right]$, we have

$$
\forall I^{\prime \prime}\left(\left(I \leq I^{\prime \prime} \Rightarrow I^{\prime} \leq I^{\prime \prime}\right) \&\left(I^{\prime \prime} \leq I \Rightarrow I^{\prime \prime} \leq I^{\prime}\right)\right)
$$

In particular, for $I^{\prime \prime}=[a, a]$, we have $I \leq I^{\prime \prime}$ and thus, we have $I^{\prime} \leq I^{\prime \prime}$, i.e., $\left[a^{\prime}, b^{\prime}\right] \leq[a, a]$. According to our description of the "possibly larger" relation, this means that $a^{\prime} \leq a$.

Similarly, for $I^{\prime \prime}=[b, b]$, we have $I^{\prime \prime} \leq I$ and thus, we have $I^{\prime \prime} \leq I^{\prime}$, i.e., $[b, b] \leq\left[a^{\prime}, b^{\prime}\right]$. According to our description of the "possibly larger" relation, this means that $b \leq b^{\prime}$.

So, $a^{\prime} \leq a \leq b \leq b^{\prime}$, which means exactly that $[a, b] \subseteq$ $\left[a^{\prime}, b^{\prime}\right]$.

Final step: it is possible to define degenerate intervals based only on the order. We already know how to define inclusion in terms of the order.

Then, similarly to the case of "necessarily larger" relation, we can define a degenerate interval $I$ as the one that does not have any subinterval different from itself:

$$
\forall J(J \subseteq I \Rightarrow J=I) .
$$

\section{Beyond Interval-VAlued Degrees: Set-VAlued DEGREES}

Set-valued degrees and hesitant fuzzy sets. In some cases, not all the values from the interval $[\underline{\mu}(x), \bar{\mu}(x)]$ are possible. In such cases, instead of an interval of possible values of degree of confidence, we have a general set of such values.
It is reasonable to require that this set be closed, i.e., that if $x_{n} \in S$ and $x_{n} \rightarrow x$, then we should have $x \in S$. Indeed, in this case, no matter how accurately we measure the degrees, the limit value $x$ will be - within this accuracy indistinguishable from some possible value $x_{n}$. Thus, we will never be able to conclude that $x$ is not possible - and thus, it is reasonable $\mathrm{t}$ consider $x$ to be possible as well.

In such cases, to describe a fuzzy property like "small", we assign, to each possible values $x$ of the corresponding quantity, a set-valued degree $S(x) \subseteq[0,1]$ describing to what extent the value $x$ satisfies the given property (i.e., to what extent $x$ is small). Such set-valued fuzzy sets are also known as hesitant sets; see, e.g., [6], [7], [9].

A natural question. For sets $S$ and $S^{\prime}$, we similarly define the above three orders:

- the "lattice-type" order

$$
\left(\forall s \in S \exists s^{\prime} \in S^{\prime}\left(s \leq s^{\prime}\right)\right) \&\left(\forall s^{\prime} \in S^{\prime} \exists s \in S\left(s \leq s^{\prime}\right)\right) ;
$$

- the "necessarily larger" order $\forall s \in S \forall s^{\prime} \in S^{\prime}\left(s \leq s^{\prime}\right)$, and

- the "possibly larger" relation $\exists s \in S \exists s^{\prime} \in S^{\prime}\left(s \leq s^{\prime}\right)$.

(One can easily check that for the case when $S$ and $S^{\prime}$ are intervals, the above formula for the "lattice-type" order leads exactly the lattice order between $S$ and $S^{\prime}$.)

A natural question is: can we extend the above results to setvalued degrees? In other words, for each of these three orders, based only on each of these three orders, can we determine one-point sets (which correspond to traditional $[0,1]$-based fuzzy degrees)?

Our answer. Our answer to this question is based on the fact whose proof is given below - that in all three definitions of the order between the sets, the relation $S \leq S^{\prime}$ is equivalent to a similar relation between their interval hulls $[S]$ and $\left[S^{\prime}\right]-$ i.e., smallest intervals containing these sets: $S \leq S^{\prime} \Leftrightarrow[S] \leq\left[S^{\prime}\right]$.

Since we have already proven that based on the order between intervals, we can reconstruct the exact fuzzy values, this means that we can also reconstruct the exact fuzzy values if we only have the order between the sets.

Preliminary analysis. To prove the desired equivalences, let us first analyze what are the corresponding interval hulls.

Each of the sets $S$ and $S^{\prime}$ is a subset of the bounded interval $[0,1]$ and is thus, bounded itself. Therefore, e.g., for the set $S$, its infimum (greatest lower bound) $a$ and its supremum (least upper bound) $b$ are both finite. Both the infimum and the supremum are limits of elements from the set $S$. Since the set $S$ is closed, these limits belong to the set $S$. So, we have $a \in S, b \in S$, and $a \leq s \leq b$ for all $s \in S$. Thus, the interval hull of the set $S$ coincides with the interval $[a, b]$ formed by its infimum $a$ and its supremum $b$.

Similarly, the interval hull $\left[S^{\prime}\right]$ of the set $S^{\prime}$ is equal to the interval $\left[a^{\prime}, b^{\prime}\right]$, where $a^{\prime}$ is the infimum of the set $S^{\prime}$ and $b^{\prime}$ is the supremum of this set. 
Proof that the order between sets is equivalent to the order between their interval hulls: case of "lattice-type" order. Let us now prove the desired equivalence for the case of the "lattice-type" oder.

Let us first assume that for every $s \in S$, there exists an element $s^{\prime} \in S^{\prime}$ for which $s \leq s^{\prime}$. Let us prove that in this case, the same property holds for the interval hulls $[S]=[a, b]$ and $\left[S^{\prime}\right]=\left[a^{\prime}, b^{\prime}\right]$, i.e., that for every point $p \in[a, b]$ there exists a value $p^{\prime} \in\left[a^{\prime}, b^{\prime}\right]$ for which $p \leq p^{\prime}$.

Indeed, as we have mentioned, the supremum $b$ of the set $S$ belongs to the set $S: b \in S$. Thus, due to our assumption, there exists a point $s^{\prime} \in S^{\prime}$ for which $b \leq s^{\prime}$. Since $p \in[a, b]$, we have $p \leq b$ and thus, $p \leq s^{\prime}$. Due to $S^{\prime} \subseteq\left[S^{\prime}\right]=\left[a^{\prime}, b^{\prime}\right]$, we conclude that $s^{\prime} \in\left[a^{\prime}, b^{\prime}\right]$. Therefore, we can take $s^{\prime}$ as the desired value $p^{\prime}$ for which $p^{\prime} \geq p$.

Similarly, we can prove that if for every $s^{\prime} \in S^{\prime}$, there exists an element $s \in S$ for which $s \leq s^{\prime}$, then the same property holds for the interval hulls $[S]=[a, b]$ and $\left[S^{\prime}\right]=\left[a^{\prime}, b^{\prime}\right]$, i.e., for every point $p^{\prime} \in\left[a^{\prime}, b^{\prime}\right]$ there exists a value $p \in[a, b]$ for which $p \leq p^{\prime}$.

Vice versa, let us now assume that for every point $p \in[a, b]$ there exists a value $p^{\prime} \in\left[a^{\prime}, b^{\prime}\right]$ for which $p \leq p^{\prime}$. Let us then prove that for every $s \in S$, there exists an element $s^{\prime} \in S^{\prime}$ for which $s \leq s^{\prime}$.

Indeed, since $S \subseteq[S]=[a, b]$, the property $s \in S$ implies that $s \in[a, b]$. Thus, due to our assumption, there exists a point $p^{\prime} \in\left[a^{\prime}, b^{\prime}\right]$ for which $s \leq p^{\prime}$. From $p^{\prime} \in\left[a^{\prime}, b^{\prime}\right]$, it follows that $p^{\prime} \leq b^{\prime}$, thus $s \leq b^{\prime}$. We have shown that $b^{\prime} \in S^{\prime}$, thus we can take $b^{\prime}$ as the desired point $s^{\prime} \in S^{\prime}$.

Similarly, we can prove that if for every point $p^{\prime} \in\left[a^{\prime}, b^{\prime}\right]$ there exists a value $p \in[a, b]$ for which $p \leq p^{\prime}$, then for every $s^{\prime} \in S^{\prime}$, there exists an element $s \in S$ for which $s \leq s^{\prime}$.

The equivalence is proven.

Proof that the order between sets is equivalent to the order between their interval hulls: case of "necessarily larger" order. Let us first prove that if we have $s \leq s^{\prime}$ for all $s \in S$ and $s^{\prime} \in S^{\prime}$, then we have $p \leq p^{\prime}$ for all $p \in[a, b]$ and $p^{\prime} \in\left[a^{\prime}, b^{\prime}\right]$.

Indeed, since $b \in S$ and $a^{\prime} \in S^{\prime}$, then, due to our assumption, we have $b \leq a^{\prime}$. Since $p \leq b$ and $a^{\prime} \leq p^{\prime}$, we thus have $p \leq p^{\prime}$.

Vice versa, if $p \leq p^{\prime}$ for all $p \in[a, b]$ and $p^{\prime} \in\left[a^{\prime}, b^{\prime}\right]$, then, since $S \subseteq[a, b]$ and $S^{\prime} \subseteq\left[a^{\prime}, b^{\prime}\right]$, we have $s \leq s^{\prime}$ for all $s \in S$ and for all $s^{\prime} \in S^{\prime}$.

The equivalence is proven.

Proof that the order between sets is equivalent to the order between their interval hulls: case of "possibly larger" order. If we have $s \leq s^{\prime}$ for some $s \in S$ and $s^{\prime} \in S^{\prime}$, then, since $S \subseteq[a, b]$ and $S^{\prime} \subseteq\left[a^{\prime}, b^{\prime}\right]$, we have $s \leq s^{\prime}$ for $s \in[a, b]$ and $s^{\prime} \in\left[a^{\prime}, b^{\prime}\right]$.

Vice versa, let us assume that $p \leq p^{\prime}$ for some $p \in[a, b]$ and $p^{\prime} \in\left[a^{\prime}, b^{\prime}\right]$. Let us prove that in this case, we have $s \leq s^{\prime}$ for some $s \in S$ and for some $s^{\prime} \in S^{\prime}$ : namely, for $s=p$ and $s^{\prime}=p^{\prime}$.

The equivalence is proven.

\section{YET ANOTHER GENERALIZATION OF INTERVAL-VALUED FUZZY SETS: FROM \\ INTERVALS (PAIRS) TO GENERAL TUPLES}

From $[0,1]$-based fuzzy degrees to interval-based and to general tuples. In the traditional $[0,1]$-based fuzzy logic, the expert's degree of certainty is described by a number from the interval $[0,1]$.

In the interval-valued fuzzy technique, the expert's degree of certainty is described by an interval $[a, b] \subseteq[0,1]$. To describe an interval, one needs to describe two numbers $a \leq b$. From this viewpoint, describing an interval is equivalent to describing a pair $(a, b)$ of degrees, a pair for which $a \leq b$. The most natural order between the intervals is a componentwise order when we say that $(a, b) \leq\left(a^{\prime}, b^{\prime}\right)$ if and only if $a \leq a^{\prime}$ and $b \leq b^{\prime}$.

Along these lines, a natural idea is to consider, for every natural number $n, n$-element tuples $(a, b, \ldots, c)$ for which $a \leq$ $b \leq \ldots \leq c$ and $(a, b, \ldots, c) \leq\left(a^{\prime}, b^{\prime}, \ldots, c^{\prime}\right)$ if and only if $a \leq a^{\prime}, b \leq b^{\prime}, \ldots$, and $c \leq c^{\prime}$.

Examples of $n$-tuples. One possible example of such tuples is the case of twin intervals, when we have an interval $[a, b]$ whose endpoints are only known with interval uncertainty, i.e., when we know the interval $[a, \bar{a}]$ of possible values of the lower endpoint $a$ and we know the interval $[\underline{b}, \bar{b}]$ of possible values of the upper endpoint $b$. In this cases, we have $\underline{a} \leq$ $\bar{a} \leq \underline{b} \leq \bar{b}$, i.e., we have, in effect, a 4-tuple.

In principle, we can consider cases in which the endpoints $\underline{a}, \bar{a}, \underline{b}$, and $\bar{b}$ are also known with interval uncertainty. In this case, we have an $n$-tuple with $n=8$. This constructions can be continued, as a result of which we can come up with $n$-tuples with larger and larger values $n$.

Another examples comes from considering interval-valued intuitionistic fuzzy degrees; see, e.g., [1]. In intuitionistic fuzzy logic, a description of the expert's confidence in a statement $S$ consists of two numbers:

- a number $a^{+}$that describes the expert's degree of certainty in $S$ and

- a number $a^{-}$that describes the expert's degree of certainty in the opposite statement $\neg S$.

These two values must satisfy a natural inequality $a^{+} \leq$ $1-a^{-}$. This description can be viewed as equivalent to considering an interval $\left[a^{+}, 1-a^{-}\right]$of possible values.

It is reasonable to consider interval-valued intuitionistic degrees, in which both degrees $a^{+}$and $a^{-}$are known with interval uncertainty. This is equivalent to considering an interval $\left[a^{+}, 1-a^{-}\right]$in which both endpoints are known with interval uncertainty - i.e., equivalent to considering 4-tuples.

A question. It is reasonable to ask: for each $n$, based on the above component-wise order between the $n$-tuples, can we reconstruct the exact fuzzy values, i.e., the tuples $(a, b, \ldots, c)$ for which $a=b=\ldots=c$ ?

Determining exact values based on the ordering between $n$-tuples. Let us now show that, based on the component- 
wise ordering between $n$-tuples, we can uniquely determine the exact values. We will show it step by step.

First, we can uniquely determine the degree $0=$ $(0,0, \ldots, 0)$ as the only degree for which $0 \leq A$ for any other degree $A=(a, b, \ldots, c)$.

Then, similarly to the interval case, we can show that the only degrees $A$ for which the set $\{B: 0 \leq B \leq A\}$ is linearly ordered are degrees of the type $A=(0, \ldots, 0, c)$ for some $c \geq 0$.

After that, we can define degrees of the type $(0, \ldots, 0, b, c)$ with $0<b$ as the degrees $A$ that satisfy the following two properties:

- the degree $A$ is not of the type $(0, \ldots, 0, c)$, and

- there exists a degree $B$ of the type $(0, \ldots, 0, c)$ for which the set $\{C: B \leq C \leq A\}$ is linearly ordered.

For a given $B$ of the type $(0, \ldots, 0, c)$ (i.e., in effect, for a given real number $c \in[0,1]$ ), we can have several degrees $A$ that satisfy these two properties. One can see that the largest of them is the degree $(0, \ldots, 0, c, c)$ corresponding to the largest possible value $b=c$.

We can then define degrees of the type $(0, \ldots, 0, b, c, c)$ with $0<b$ as the degrees $A$ that satisfy the following two properties:

- the degree $A$ is not of the type $(0, \ldots, 0, c, c)$, and

- there exists a degree $B$ of the type $(0, \ldots, 0, c, c)$ for which the set $\{C: B \leq C \leq A\}$ is linearly ordered.

For a given $B$ of the type $(0, \ldots, 0, c, c)$ (i.e., in effect, for a given real number $c \in[0,1]$ ), we can have several degrees $A$ that satisfy these two properties. One can see that the largest of them is the degree $(0, \ldots, 0, c, c, c)$ corresponding to the largest possible value $b=c$.

Continuing along the same lines, we eventually arrive at the description of the degrees of the type $(c, \ldots, c)$, i.e., at the desired description of the $n$-tuples that correspond to exact $([0,1]$-based) fuzzy degrees. The desired possibility to determine exact fuzzy values based on ordering between the $n$-tuples is thus proven.

\section{CONCLUSIONS}

In many application areas, expert knowledge is very important. It is therefore desirable to add this knowledge to the knowledge base that computers use when making corresponding conclusions and decisions. One of the challenges in representing expert knowledge in a computer is that experts often formulate their knowledge by using imprecise ("fuzzy") words from natural language, such as "small". One of the main objectives of fuzzy techniques is to translate such "fuzzy" knowledge into a precise computer-understandable statement. For this purpose, in the traditional [0,1]-based fuzzy logic, every fuzzy property like "small" is described by a function $\mu(x)$ that assigns, to each possible value $x$ of the corresponding quantity, the expert's degree of confidence that this value $x$ satisfies the given property (e.g., the degree to which $x$ is small).

This degree is elicited by an expert - e.g., by asking the expert to mark the corresponding degree on a scale from 0 to 1 (alternatively, we can ask the expert to mark it on a scale from 0 to 10 , and then divide the resulting mark by 10). The problem with such elicitation is that since the expert is unable to describe his/her opinion in precise terms, this expert will also be probably unable to describe his/her degree of uncertainty by a precise number. A more realistic way of describing the expert's degree of certainty is to allow the expert to mark the whole range $[\mu(x), \bar{\mu}(x)]$ of numbers instead of a single number.

In some cases, the expert can have an exact number $\mu(x)$ as a mark. In these cases, the intervals becomes a single point $[\mu(x), \mu(x)]$. Such intervals are known as degenerate.

On the set of such interval-valued degrees of certainty, we can describe a natural order: e.g., we can say that the degree $[a, b]$ describe a lower level of certainty that the degree $\left[a^{\prime}, b^{\prime}\right]$ if $a \leq a^{\prime}$ and $b \leq b^{\prime}$. A natural question is then: if we only have this order, can we use this order to determine degenerate intervals - which correspond to exact fuzzy values?

In this paper, we show that such a determination is always possible - and not only for the above-described order, but also for several other reasonable orders between intervals. We also show that this result can be extended beyond interval-valued fuzzy degrees - e.g., to set-valued ones.

\section{ACKNOWLEDGMENTS}

This work was supported in part by the National Science Foundation grants HRD-0734825 and HRD-1242122 (CyberShARE Center of Excellence) and DUE-0926721, and by an award "UTEP and Prudential Actuarial Science Academy and Pipeline Initiative" from Prudential Foundation.

The authors are thankful to the anonymous referees for valuable suggestions.

\section{REFERENCES}

[1] K. Atanassov, Intuitionistic Fuzzy Sets: Theory and Applications, Springer-Verlag, Berlin, Heidelberg, 1999.

[2] G. Klir and B. Yuan, Fuzzy Sets and Fuzzy Logic, Prentice Hall, Upper Saddle River, New Jersey, 1995

[3] J. M. Mendel, Uncertain Rule-Based Fuzzy Logic Systems: Introduction and New Directions, Prentice-Hall, Upper Saddle River, 2001.

[4] J. M. Mendel and D. Wu, Perceptual Computing: Aiding People in Making Subjective Judgments, IEEE Press and Wiley, New York, 2010.

[5] H. T. Nguyen and E. A. Walker, A First Course in Fuzzy Logic, Chapman and Hall/CRC, Boca Raton, Florida, 2006.

[6] M. Rodríguez, L. Martínez, V. Torra, Z. S. Xu, and F. Herrera, "Hesitant fuzzy sets: state of the art and future directions", International Journal of Intelligent Systems, 2014, Vol. 29, pp. 495-524.

[7] V. Torra, "Hesitant fuzzy sets", International Journal of Intelligent Systems, 2010, Vol. 25, pp. 529-539.

[8] C. Wagner, S. Miller, J. M. Garibaldi, D. T. Anderson, and T. C. Havens, "From interval-valued data to general type-2 fuzzy sets", IEEE Transactions on Fuzzy Systems, 2015, Vol. 23, No. 2, pp. 248-269.

[9] Z. Hu, Hesitant Fuzzy Sets Theory, Springer Verlag, Berlin, Heidelberg, New York, 2014

[10] L. A. Zadeh, "Fuzzy sets", Information and Control, 1965, Vol. 8, pp. 338-353. 